\title{
Comparison of Epidemiological, Clinical, Laboratory and Radiological Features of Hospitalized Diabetic and Non-Diabetic Patients With Pulmonary Tuberculosis at Razi Hospital in Ahvaz
}

\author{
Seyed Mohammad Alavi ${ }^{1,}$; Mohammad Mehdi Khoshkho $^{2}$; Shokrolah Salmanzadeh ${ }^{3}$; Me- \\ hdi Eghtesad ${ }^{3}$ \\ ${ }_{2}^{1}$ Health institute,Infectious and Tropical Diseases Research Center, Jundishapur University of Medical Sciences, Ahvaz, IR Iran \\ ${ }_{2}^{2}$ Infectious Diseases Department, Razi Hospital, Medical College, Jundishapur University of Medical Sciences, Ahvaz, IR Iran \\ ${ }^{3}$ Khuzestan Health Center, Jundishapur University of Medical Sciences, Ahvaz, IR Iran \\ ${ }^{*}$ Corresponding author: Seyed Mohammad Alavi, Health institute,Infectious and Tropical Diseases Research Center, Jundishapur University of Medical Sciences, Ahvaz, Iran. Tel: +98- \\ 6113387724, Fax: +98-6113335396, E-mail: alavi.seyedmohammad@yahoo.com
}

Received: June 20, 2013; Revised: September 22, 2013; Accepted: October 5, 2013

\begin{abstract}
Background: Diabetes mellitus (DM) due to suppressive effect on cellular immunity can impact on progression of tuberculosis(TB).
Objectives: The aim of this study was to investigate the impact of DM on the epidemiological, clinical and para clinical aspects of pulmonary TB.

Patients and Methods: The information of 148 admitted pulmonary TB patients in infectious ward of Razi hospital in Ahvaz from 2009 to 2010 was extracted from their medical files. The patients were divided into two groups as TB with DM ( $n=36)$ and TB without DM $(n=112)$. The related data on epidemiology, signs, symptoms, radiology and sputum smear examination in both groups were compared in SPSS 16 by using chi squared test.

Results: The mean age of TB with DM patients was higherTB without DM patients ( $56.6 \pm 12.7 \mathrm{vs.} 44.8 \pm 18.3$; respectively, $\mathrm{P}=0.006$ ). Whereas cough, night sweating, fever and weigh loss was not statistically different, sputum, hemoptysis and dyspnea was more prominent in TB with $\operatorname{DM}(69.4 \%, 33.4 \%, 44.5 \%$ vs. 36.6\%, 9.8\%, 20.5\%; $\mathrm{P}=0.005, \mathrm{P}=0.001, \mathrm{P}=0.005$, respectively). In chest $\mathrm{x}$-ray, cavitation and reticulonodular pattern was more frequent in TB with $\mathrm{DM}(55.5 \%, 22.2 \% \mathrm{vs} .31 .2 \%, 8 \%-\mathrm{P}=0.008, \mathrm{P}=0.02$, respectively). The rate of sputum smear positivity in TB with DM and TB without DM was $66.6 \%$ and $47.3 \%$, respectively $(\mathrm{P}=0.03)$.

Conclusions: According to the results of this study, in approach to every DM cases suffering of respiratory symptoms such as productive cough, hemoptysis and dyspnea in association with cavitation or miliary mottling in chest x-ray, pulmonary TB should be considered at the top of the differential diagnosis list.
\end{abstract}

Keywords:Pulmonary tuberculosis; Diabetes Mellitus; Epidemiology; Clinical Features; Radiology

\section{Background}

Despite the Directly Observed Treatment Short course (DOTS) strategy, tuberculosis (TB) is still a major public health problem in the world (1). According to the World Health Organization (WHO), about 8 to 8 million people annually are diagnosed with $\mathrm{TB}$, the majority of them occur in developing countries $(2,3)$. Diabetes is a debilitating disease that impairs the cellular immune system and provides the ground for TB. The WHO estimates that by 2030 , the population of diabetics is increasing and reaches about 316 million people, most of them are living in developing countries (4-6).

Diabetes mellitus (DM) is a common chronic metabolic disorder that affects mainly the country's elder population. According to the Center for Disease Control (CDC), the prevalence of DM in the United States is approximately $8 \%$ (7). The leukocyte dysfunction and reduction of serum bactericidal activity in DM patients increases the risk of TB infection $(8,9)$. Several prospective and retrospective studies have shown a relationship between TB and DM. Patients with DM are at increased risk of pulmonary TB (1017). Knowing the epidemiological, clinical, laboratory and radiological aspects of patients with tuberculosis helps involved practitioners in rapid diagnosis and initiating appropriate treatment. Prompt and appropriate treatment is the most effective way to prevent of treatment failure, relapse, or emergence of multi drug resistant TB (MDR$\mathrm{TB}$ ) which today is the main problems in fighting against $\mathrm{TB}$ (3). Because the differences in the immune response in diabetics and non-diabetic infected hosts ,epidemiological, clinical, radiological, laboratory tests results and response to treatment varies in these patients $(8,9)$.

Tuberculosis in older people with weak immunity is associated with less cough and fever in compare with younger individuals while, pleural effusion and cavitation are more common in patients with normal immune responses (3). To the best our knowledge, such study in

Copyright (c) 2014, Ahvaz Jundishapur University of Medical Sciences; Published by Kowsar Corp. This is an open-access article distributed under the terms of the Creative Commons Attribution License, which permits unrestricted use, distribution, and reproduction in any medium, provided the original work is properly cited. 
diabetic patients with pulmonary tuberculosis has not been performed in Khuzestan - a province with a population of over 4.5 million. Studies in this field are very limited in the country (18). Since the majority of deaths in patients with pulmonary tuberculosis occur in hospitalized patients, thus, hospital-based studies provide useful information on various aspects of the disease.

\section{Objectives}

Considering that knowing this information is necessary to control TB, this study aimed to compare the epidemiological characteristics, clinical, laboratory and radiological findings in patients with pulmonary TB in diabetic and non-diabetic patients.

\section{Patients and Methods}

In this retrospective study, all cases of pulmonary $\mathrm{TB}$, hospitalized in Razi hospital during two years (2009 and 2010) were reviewed. To find more information, complete data and matching the data with health file of patients, TB documents in Khuzestan health center were also studied. Razi hospital in Ahvaz is the referral center for almost all cases of pulmonary TB, therefore, we assumed the above cases as $s$ representative of all TB cases in Khuzestan. Patients' data on demographics, residential area, habits, past medical history, laboratory and sputum microscopy analysis, as well as clinical signs and symptoms, radiological findings, the patient's condition of diabetes (self-referred, under treatment of oral anti-diabetic medication and fasting blood sugar levels), receiving anti-TB drugs, TB treatment response and other relevant information were extracted from patient records. Patients were divided into two groups; a group of patients with pulmonary tuberculosis and with diabetes mellitus called as DM \& PTB, and other group of patients with pulmonary tuberculosis but without diabetes mellitus called as Non-DM \& PTB.

Study inclusion criteria were as follow: patients who had pulmonary tuberculosis, based upon the national anti-TB program (NTP). Pulmonary TB: at least two tests of positive sputum smear acid-fast test or one positive sputum smear acid-fast and a chest radiograph consistent with TB, smear-negative pulmonary tuberculosis; at least 3 sputum tests negative for acid-fast bacilli (AFB) but positive radiographic or a doctor's decision on anti-TB treatment in the presence of clinical signs and symptoms consistent with TB and age older than 15 years. In additions, the exclusion criteria were cases with no state of sputum smear analysis, cases with incomplete information and non-diabetic patients but, with other underlying diseases that may influence the immune status. Patient's data in both groups were analyzed in SPSS-16 using Chi-square test and Fisher exact tests. Differences at P value of less than 0.05 were considered significant.

\section{Results}

From 169 cases of PTB, 5 patients were excluded due to incomplete data, 8 due to defects in the state of sputum smear results, and 8 due to HIV infection. Of the remaining 148 patients, 36 patients had diabetes mellitus. Mean age in the DM \& PTB and Non-DM \& PTB group were $(\mathrm{n}=$ 36), $56.6 \pm 12.7(n=112)$ was $44.8 \pm 18.3$ years, respectively. As shown in Table 1, prevalence of pulmonary tuberculosis among older than 60-years diabetic patients have no significant difference with those below 60 years of age, whereas, the mean age in diabetic's cases was higher than in non-diabetics. Although more males than females are diagnosed with PTB, but this difference was not significant. Also, while TB prevalence is higher in urban areas than in rural ones, he difference is not significant. Clinical signs and symptoms in both groups are shown in Table 2. Although symptoms such as cough, night sweat, fever, and weight loss in both groups exist, but, sputum production, hemoptysis, and dyspnea in patients with diabetes were more prevalent than in non-diabetic patients. Radiological features in non-diabetic and diabetic patients are shown in Table 3.

There is a similar frequency of infiltration and upper lobe opacity in both groups, but there is a higher frequency of the cavity lesions and reticulunodular pattern in diabetic patients comparing with non-diabetic patients. In diabetic and non-diabetic patients, cell blood count (CBC) was normal (normal range: 7000-10000) in 108 patients $(96.4 \%), 31$ patients $(86.1 \%)$, which was not statistically significant $(P>0.05)$. Mean value of erythrocyte sedimentation rate (ESR) in diabetic and non-diabetic patients were respectively 49.6 and $53.3 \mathrm{~mm} / \mathrm{h}$, which was not statistically significant $(\mathrm{P}>0.05)$. In diabetic patients, sputum smear was positive for AFB in 24 (66.6\%) patients, while in non-diabetic patients it was positive in $53(47.3 \%)$ patients, which was statistically significant $(\mathrm{P}=$ 0.03). Number of MDR-TB in the non-diabetic group was 2 , and in diabetic group was 4 , although statistically was not comparable, but regarding to the country rate and expected rate in NTP (2\%) and percent in these patients (5.5\% vs. $3.5 \%)$ is considerable.

Table 1. Demographic Characteristics of Diabetic and nonDiabetic Patients ${ }^{\mathrm{a}, \mathrm{b}}$

\begin{tabular}{|c|c|c|c|}
\hline Variables & DM \& PTB $(n=36)$ & $\begin{array}{l}\text { Non-DM \& PTB } \\
\quad(n=112)\end{array}$ & PValue \\
\hline Age, y & & & 0.20 \\
\hline$>60$ & $27(75)$ & $93(83)$ & \\
\hline$<60$ & $9(25)$ & $19(17)$ & \\
\hline Gender & & & 0.32 \\
\hline Male & $20(55.5)$ & $69(61.6)$ & \\
\hline Female & $16(44.5)$ & $43(38.4)$ & \\
\hline Residential area & & & 0.24 \\
\hline Urban & $24(66.6)$ & $61(54.4)$ & \\
\hline Rural & $12(43.4)$ & 51 & \\
\hline
\end{tabular}


Alavi SM et al.

Table 2. Clinical Signs and Symptoms in Diabetic and non-Diabetic Patients ${ }^{\mathrm{a}, \mathrm{b}}$

\begin{tabular}{lccc}
\hline Variables & DM \& PTB $(\mathbf{n}=\mathbf{3 6})$ & Non-DM \& PTB $(\mathbf{n}=\mathbf{1 1 2})$ & PValue \\
\hline Cough & $34(94.4)$ & $106(94.6)$ & 0.62 \\
Sputum production $^{\mathrm{c}}$ & $25(69.4)$ & $41(36.6)$ & 0.0005 \\
Hemoptysis $^{\mathrm{C}}$ & $12(33.4)$ & $11(9.8)$ & 0.001 \\
Night sweating & $20(55.5)$ & $59(52.7)$ & 0.45 \\
Fever & $22(61.1)$ & $63(56.2)$ & 0.37 \\
Dyspnea $^{\mathrm{C}}$ & $16(44.5)$ & $23(20.5)$ & 0.005 \\
Weight loss & $17(47.2)$ & $48(42.8)$ & 0.39 \\
\hline
\end{tabular}

a Abbreviations: DM \& PTB, diabetes mellitus and with pulmonary tuberculosis.

$\mathrm{b}$ Data are presented as No.(\%).

C Statistically significant

Table 3. Radiological Findings of Diabetic and non-Diabetic Patients a,b

\begin{tabular}{lccc}
\hline Variables & Non-DM \& PT $(\mathbf{n}=\mathbf{1 1 2})$ & DM \& PTB $(\mathbf{n}=\mathbf{3 6})$ & P Value \\
\hline Upper lobe opacity & $24(66.6)$ & $59(52.7)$ & 0.09 \\
Plural effusion $^{\text {Cavitation }}{ }^{\mathrm{C}}$ & $3(8.3)$ & $16(14.3)$ & 0.26 \\
Nodular infiltration (military mottling) $^{\mathrm{C}}$ & $20(55.5)$ & $35(31.2)$ & 0.008 \\
\hline
\end{tabular}

a Abbreviations: DM \& PTB, diabetes mellitus and with pulmonary tuberculosis

${ }^{b}$ Data are presented as No. (\%)

c Statistically significant

\section{Discussion}

Although some epidemiological features of pulmonary tuberculosis such as gender, age and residential area are similar in both diabetic and non-diabetic patients, but in clinical point of view, radiological and laboratory features were more dominant in diabetic pulmonary tuberculosis-infected patients comparing with non-diabetics patients. In dealing with patients suspected of having $\mathrm{TB}$, these differences should be considered. These results are consistent with other studies (10,11, 15-19). Although, the incidence of TB in people over 60 years and under 60 years showed no significant difference; however, the mean age of patients with pulmonary tuberculosis in diabetic patients was higher than non-diabetic patients. This is justified because, firstly, old TB infected persons are at in increased risk of TB reactivation; secondly, the incidence of diabetes is higher in the older age.

We cannot easily say that the incidence of tuberculosis in elderly diabetics is higher than aged non-diabetics. To demonstrate this, we first need to compare non-TB diabetic and non-diabetic cases for age. Baghaei et al. (18) in their study in Masih Daneshvari Hospital (the country's referral center) in Tehran, Iran, showed that the mean age of diabetic patients with pulmonary tuberculosis is a little more than non-diabetic TB patients ( 57.8 vs 55.2 years). Ezung et al. have also shown that the mean age of TB patients with diabetes is higher than non-diabetic TB patients (11). This study shows that patients in rural area have higher prevalence of diabetes and TB comparing to rural areas. Suburbs, where people live in crowd environments greater chance of exposure to infectious TB patients may causes great number of AFB in the air. Because of the limited studies in this field, comparing the results of this study with other studies in other areas of the country is not possible. Stevenson et al., has also pointed out a higher incidence of pulmonary tuberculosis in diabetic patients in urban areas than in rural areas (15).

The present study revealed that cough, fever and night sweats in diabetic patients are the same in non-diabetic patients, but the sputum production, hemoptysis, and dyspnea are observed in the diabetics more frequent than in non-diabetics. Baghaei et al. has demonstrated that anorexia, dyspnea and hemoptysis in diabetes were higher than in non-diabetes but, cough and sputum production in both groups were similar (18). In general, the results of the clinical findings in this study are concordant to the results of other studies conducted in other regions $(15,17)$. It seems that diabetes mellitus patients, because of diabetes-induced immunodeficiency, are incapable of inhibiting the progression to disease, therefore, disease progress more rapidly and the blood vessel wall and pleural involvement results in hemoptysis and dyspnea.

These results are in agreement with previous studies in medical literature $(3,10,11,13,17)$. In this study the radiographic findings, such as upper lobe opacities and pleural effusions, are similar in both groups, but cavitation and nodular infiltration (miliary mottling) pattern are 
more observed in diabetic compared with non-diabetic patients (3). Regarding the fact that the inflammatory response is suppressed in immunocopromised host, frequency of cavitary lesions in non-diabetic individuals with normal immunity is conceivable. However miliary pattern in patients with diabetes can be explained for of the spread of disease because of cellular immunity impairment. However, because the study was retrospective, it is not clear exactly how their diabetes was controlled and how they were their immune status.

Future studies are needed to clarify this issue with the substantial sample size and the level of cellular immunity. Except in Baghaei et al study (18) with similar radiological findings in both groups (except in cavitation), no other studies were found regarding this issue, addressing our country. They reported that diabetic patients had a higher prevalence of typical presentations along with cavitary lesions (18). But in the studies of other countries, the prevalence of cavities in chest radiography of diabetic patients with pulmonary tuberculosis has been suggested $(10,11,17)$.

In this study, sputum AFB positivity in diabetes was more prevalent than in non-diabetic patients. In other words, the chances of isolating the tuberculosis bacillus in the sputum of diabetic TB patients are more than nondiabetic patients. These findings are in agreement with other studies in this subject (15), because these patients are associated with increased sputum production, and also because of more cavitation and tissue immune inhibition, the possibility of access to further sputum containing bacilli exist. In Stevenson et al. study, higher rates of sputum positivity have been reported in diabetic patients (15). In other comorbidities, such as the elderly or patients with advanced AIDS, tuberculosis bacillus isolation level is higher than tuberculosis patients with normal immunity (3). In this study, based on limited number of drug resistance cases, multidrug-resistant TB cases cannot be discussed, however, the increasing number of patients with MDR-TB (comparing with NTP limit) has alarmed in diabetes and further prospective studies in this field is recommended. Bashar et al., has also warned the importance of MDR-TB among diabetic individuals (16).

\subsection{Study strength and limitation}

To the best our knowledge, till now, there is no similar study in the province, and studies in this field in Iran are very limited, so this study can be considered as a new work. The study design is retrospective and is limited to only one hospital. Since our hospital is the only referral infectious diseases center in Ahvaz, this limitation can be justified. Another limitation in this study is the small number of diabetic TB patients. Future population-based studies are needed to generalize these results to the whole community in the region.

Epidemiology of tuberculosis in both diabetic and nondiabetic patients is similar. Although the signs and symptoms of TB disease in diabetic patients are the same with non-diabetic ones, but sputum production, hemoptysis, and dyspnea are more prevalent in diabetics. Chest radiography in diabetic patients is a useful diagnostic modality for detecting advanced lesions such as cavity and miliary lesions. According to the results of this study, in approach to every DM case presenting with respiratory symptoms such as productive cough, hemoptysis and dyspnea in association with cavitation or reticulonodular pattern in chest x-ray, pulmonary TB should be considered at the top of the differential diagnosis list.

\section{Acknowledgements}

We greatly thank the staff of Infectious Diseases Research Center, Razi Hospital and Khuzestan Health Center affiliated to Jundishapur University of Medical Sciences for their kindly cooperation in the research and conducting the studies.

\section{Authors' Contributions}

Study concept and design, Seyed Mohammad Alavi; Analysis and interpretation of data, Seyed Mohammad Alavi, Shokrolah Salmanzadeh, and Mehdi Eghtesad; Drafting of the manuscript, Seyed Mohammad Alavi; Critical revision of the manuscript for important intellectual content, Seyed Mohammad Alavi, Mohammad Mehdi Khoshkho, and Shokrolah Salmanzadeh; Statistical analysis, Mehdi Eghtesad and Mohammad Mehdi Khoshkho.

\section{Funding/Support}

This study was supported by Infectious and Tropical Research Center, Jundishapur University of Medical Sciences.

\section{References}

1. Hassan Zadeh J, Nasehi M, Rezaianzadeh A, Tabatabaee H, Rajaeifard A, Ghaderi E. Pattern of reported tuberculosis cases in iran 2009-2010. Iran J Public Health. 2013;42(1):72-8.

2. Raviglione MC, Snider DE, Jr, Kochi A. Global epidemiology of tuberculosis. Morbidity and mortality of a worldwide epidemic. JAMA. 1995;273(3):220-6.

3. Fitzgerald D, Hass W. Mycobacterium tuberculosis. In: Mandell G, Bennett J, Raphael D editors. Principles and Practice of Infectious Diseases.. Philadelphia: Churchill Livingstone; 2010. pp. 3129-51.

4. Wild S, Roglic G, Green A, Sicree R, King H. Global prevalence of diabetes: estimates for the year 2000 and projections for 2030 . Diabetes Care. 2004;27(5):1047-53.

5. Deerochanawong C, Ferrario A. Diabetes management in Thailand: a literature review of the burden, costs, and outcomes. Global Health. 2013;9:11.

6. World Health Organization.. Non-communicable diseases country profile. Geneva Switzerland: WHO; 2010. Available from: http:/| www.who.int/nmh/publications/ncd_profiles2011/en/.

7. Esteghamati A, Ashraf H, Khalilzadeh O, Rashidi A, Mohammad $\mathrm{K}$, Asgari $\mathrm{F}$, et al. Trends of diabetes according to body mass index levels in Iran: results of the national Surveys of Risk Factors of Non-Communicable Diseases (1999-2007). Diabet Med. 2010;27(11):1233-40

8. Delamaire M, Maugendre D, Moreno M, Le Goff MC, Allannic H, Genetet B. Impaired leucocyte functions in diabetic patients. Diabet Med.1997;14(1):29-34. 


\section{Alavi SM et al.}

9. McMahon MM, Bistrian BR. Host defenses and susceptibility to infection in patients with diabetes mellitus. Infect Dis Clin North Am. 1995;9(1):1-9.

10. Perez-Guzman C, Torres-Cruz A, Villarreal-Velarde H, Vargas MH. Progressive age-related changes in pulmonary tuberculosis images and the effect of diabetes. Am J Respir Crit Care Med. 2000;162(5):1738-40.

11. Ezung T, Devi NT, Singh NT, Singh TB. Pulmonary tuberculosis and diabetes mellitus--a study.J Indian Med Assoc. 2002;100(6):376.

12. Jeon CY, Murray MB. Diabetes mellitus increases the risk of active tuberculosis: a systematic review of 13 observational studies. PLoS Med. 2008;5(7).

13. Alavi SM, Salami N. The causes of death among patients with tuberculosis in Khuzestan, Iran. PakJ Med Sci. 2008;24(2):217-20.

14. Duangrithi D, Thanachartwet V, Desakorn V, Jitruckthai P, Phojanamongkolkij K, Rienthong S, et al. Impact of diabetes mellitus on clinical parameters and treatment outcomes of newly di- agnosed pulmonary tuberculosis patients in Thailand. Int J Clin Pract. 2013;67(11):1199-209.

15. Stevenson CR, Forouhi NG, Roglic G, Williams BG, Lauer JA, Dye C et al. Diabetes and tuberculosis: the impact of the diabetes epidemic on tuberculosis incidence. BMC Public Health. 2007;7:234.

16. Bashar M, Alcabes P, Rom WN, Condos R. Increased incidence of multidrug-resistant tuberculosis in diabetic patients on the Bellevue Chest Service, 1987 to 1997. Chest. 2001;120(5):1514-9.

17. Jabbar A, Hussain SF, Khan AA. Clinical characteristics of pulmonary tuberculosis in adult Pakistani patients with co-existing diabetes mellitus. East Mediterr Health J. 2006;12(5):522-7.

18. Baghaei P, Tabarsi P, Abrishami Z, Mirsaeidi M, Faghani YA, Mansouri SD. Comparison of Pulmonary TB Patients with and without Diabetes Mellitus Type II. Tanaffos. 2010;9(2):13-20.

19. Sreeramareddy CT, Panduru KV, Menten J, Van den Ende J. Time delays in diagnosis of pulmonary tuberculosis: a systematic review of literature. BMC Infect Dis. 2009;9:91. 